\title{
DISTINCT ASPECTS OF PAIN CATASTROPHIZING ACCORDING TO CHRONIC
}

PAIN SYNDROMES

\author{
Maxciel Zortea ${ }^{1,2}$, Luciana da Conceição Antunes ${ }^{1,2}$, Joice Dickel Segabinazi ${ }^{1,2,3}$, \\ Gerardo Beltran Serrano ${ }^{1,2}$, Jéssica Lorenzzi Elkfury ${ }^{1,2}$, Fabiana Carvalho ${ }^{1,2}$, \\ Vinicius Santos ${ }^{1,2}$, Wolnei Caumo ${ }^{1,2}$
}

\footnotetext{
Clin Biomed Res. 2018;38(1):42-49

1 Laboratório de Dor \& Neuromodulação, Hospital de Clínicas de Porto Alegre (HCPA). Porto Alegre, RS, Brasil.

2 Programa de Pós-Graduação em Medicina: Ciências Médicas, Faculdade de Medicina, Universidade Federal do Rio Grande do Sul (UFRGS). Porto Alegre, RS, Brasil.

3 Pontifícia Universidade Católica do Rio Grande do Sul (PUCRS). Porto Alegre, RS, Brasil.

Corresponding author:

Maxciel Zortea

max.zortea@gmail.com

Hospital de Clínicas de Porto Alegre (HCPA)

Rua Ramiro Barcelos, 2350.

90035-903, Porto Alegre, RS, Brasil.
}

\section{ABSTRACT}

Introduction: Catastrophizing is the tendency to magnify the threat value of pain and has been associated with measures of physical and psychological disability among individuals with several pain conditions. The aim of this study was to investigate whether pain catastrophizing is differentially associated with distinct pain syndromes.

Methods: This is a cross-sectional study including 158 patients (40 with fibromyalgia, 25 with myofascial pain syndrome, 33 with chronic tensional type headache, 33 with endometriosis, and 27 with knee osteoarthritis) and 93 healthy subjects. The recruitment procedure occurred in concurrence with randomized controlled trials. Participants answered the following instruments: Brazilian Portuguese Pain-Catastrophizing Scale, Beck Depression Inventory II, State-Trait Anxiety Inventory, Pittsburgh Sleep Quality Index, visual analogue scale for pain, as well as a sociodemographic questionnaire.

Results: For the total pain catastrophizing score, patients with endometriosis had significantly more catastrophizing thoughts than knee osteoarthritis $(p<0.05)$. Healthy participants had lower scores than any clinical group. More interestingly were the dimensions of pain catastrophizing, which showed significant differences in more than two groups, especially magnification scores, since these scores were able to discriminate, in a particular way, the majority of clinical samples from each other. We also observed differences between rumination, magnification and helplessness scores in all groups, suggesting that the characteristics of pain catastrophizing are distinct according to the pain disorder.

Conclusions: The results suggest that dimensions of pain catastrophizing differ between pain syndromes. Therefore, it is important that researchers and clinicians focus on cognitive and emotional aspects of pain perception to have more successful interventions.

Keywords: Chronic pain; catastrophizing; cross-sectional study

Pain is a multidimensional sensory experience that is intrinsically unpleasant and associated with hurting and soreness ${ }^{1}$. Chronic pain is a maladaptive neuroplasticity process that manifests itself in many different pathological changes in the nervous system function, affecting $30 \%$ of the worldwide population ${ }^{2}$. Chronic pain reduces quality of life and causes an expressive social and economic burden. Widespread chronic pain has an estimated prevalence of $14.2 \%{ }^{3}$.

It is well established that central sensitization phenomenon involves impaired functioning of brain-orchestrated descending inhibitory mechanisms and can alter pain facilitatory pathways ${ }^{4,5}$, causing changes in sensory processing centers of the brain ${ }^{4}$. These neuroplastic changes can modify behavioral and emotional aspects when one is experiencing chronic pain ${ }^{6}$, being associated with clinical and psychological symptoms ${ }^{7}$. 
It has been evidenced that pain may play an important role in the development of negative emotional reactions, such as temporary or chronic anger, depression, loneliness, and anxiety. These emotions can modify the subjective experience of pain, amplifying the pain signaling process. Chapman and Nakamura ${ }^{8}$ described negative emotions as one of the most important characteristics of pain. Furthermore, some psychological factors, such as somatization, helplessness, and catastrophizing, might exacerbate chronic pain conditions and worsen prognosis ${ }^{9-11}$.

The concept of catastrophizing was first described nearly 40 years ago and aims to define the tendency to magnify the threat value of pain and to feel helpless in this context associated with an inability to inhibit pain-related thoughts in anticipation of, during or following a painful situation ${ }^{12,13}$. Consequently, it has been suggested that catastrophizing is a stable, person-based trait ${ }^{14,15}$ that probably compromises therapeutic response and prognosis. Catastrophizing related to pain can be measured by the Pain Catastrophizing Scale (PCS), which allows a prompt identification of individuals at risk for psychological consequences that may need further psychosocial evaluation. The PCS assesses three dimensions of catastrophizing in response to pain: 1) rumination, 2) magnification, and 3 ) helplessness ${ }^{14,16}$. The PCS has been broadly used in research and clinical practice. These studies have shown that catastrophizing is significantly correlated with the patient's subjective experiences on the severity of pain and his or her disability due to pain. Besides, our group has reported the relationship between cortical excitability and pain catastrophizing, suggesting that catastrophizing responses may be related to neurophysiological mechanisms associated with chronic pain, since glutamatergic activity was associated with mechanisms underlying pain catastrophizing ${ }^{17}$. Altogether, the findings above emphasize the importance of conceptualizing catastrophizing in specific pain syndromes. Nevertheless, different profiles of pain catastrophizing remains unclear. Collectively,$^{18}$ suggest that catastrophizing refers to numerous negative pain-related thoughts, including magnification of the severity of a pain perception, rumination thoughts related to pain, and helpless thoughts related to worrying about the worst possible consequences of pain.

Thus, the aim of this exploratory study was to investigate whether pain catastrophizing scores are differentially associated with distinct pain syndromes. To address this question we administered the PCS and examined its relation to pain syndrome, depression, anxiety, sleep quality, and levels of pain.

\section{METHODS}

The protocol was reviewed and data collection was approved by the Hospital de Clínicas de Porto Alegre Research Ethics Committee under protocol numbers: 10-0555, 14-0369, 14-0092, 10-0380, and 11-0013, according to the sample recruited. All patients provided written informed consent prior to their participation in this study.

\section{Design Overview, Setting, and Participants}

For this cross-sectional study, a total of 158 patients were determined to be eligible and agreed to participate: 40 with fibromyalgia (FM), 29 with myofascial pain syndrome (MPS), 33 with chronic tensional type headache (CTTH), 33 with endometriosis, and 27 with osteoarthritis (OA), in addition to 93 healthy volunteers. Chronic pain subjects recruited from local community care units, from an institutional chronic pain clinic, by referrals from other hospital units, and by phone and newspaper were included in the study. The recruitment procedure occurred in concurrence with some randomized controlled trials (RCTs) run at the Hospital de Clínicas de Porto Alegre, registered at https://clinicaltrials.gov, including studies with chronic pain disorders such as FM (NCT02041455, NCT01804097), MPS (NCT01964729), CTTH (NCT01954277), and knee osteoarthritis (KOA) (NCT01747070, NCT01855958).

Healthy volunteers were recruited from the general population by public postings. A standard screening questionnaire was developed in order to assess inclusion criteria. Eligible healthy control subjects had to be free of any acute or chronic pain; without recent use of analgesics, corticosteroids or psychotropic medications; and without any rheumatologic, psychiatric, or neurological disorders. Besides, healthy control volunteers were excluded if they reported alcohol or psychotropic substance abuse 6 months preceding the screening.

\section{Diagnosis}

A highly skilled physician trained to perform chronic pain assessment and treatment provided all diagnoses in the RCTs using standardized evaluation criteria. The diagnostic criteria for MPS included regional pain, normal neurologic examination, the presence of trigger points, taut bands, tender points, and pain characterized as "dull", "achy", or "deep". OA diagnoses were made according to the clinical and radiographic criteria of the American College of Rheumatology ${ }^{19}$. Also, all OA patients completed the Western Ontario and McMaster universities OA index (WOMAC), a validated measure of OA-related patient-reported symptoms and perceived disability ${ }^{20}$. CTTH diagnosis 
was made using the 2004 International Headache Society criteria ${ }^{21}$. FM diagnosis was made according to the American College of Rheumatology criteria for $\mathrm{FM}^{22}$. In all RCTs, patients were included if they had experienced pain scored as visual analog scale (VAS) $>40 \mathrm{~mm}$ (which is compatible to moderate or severe pain). Additionally, the pain needed to be associated with disability, as assessed by an affirmative answer to dichotomous questions (yes/no) of a structured questionnaire. Queries inquired if their pain had interfered with work, enjoyable activities, responsibilities at home, relationships, personal goals, thinking clearly, problem solving, concentration, or recall. Patients with rheumatologic or neurologic diseases (e.g.: stroke or Parkinson's disease), surgical procedure on the pain-related areas in the prior 6 months, habitual use of corticosteroids, or any other uncompensated chronic pathology were excluded. Additionally, patients with any malignancy diagnosis, with HIV were excluded, as well as those who were illiterate.

\section{Self-report Variables \\ Sociodemographic data}

After signing informed consent, subjects were asked to complete a sociodemographic questionnaire that assessed variables including age, gender, work status due to pain, and educational level.

\section{Pain assessment and psychological variables}

The Brazilian Portuguese Pain-Catastrophizing scale (BP-PCS) ${ }^{23}$ was used to measure catastrophizing thoughts related to pain. This is a self-administered questionnaire composed by 13 items that evaluates self-reported catastrophizing thoughts, feelings, and behaviors when one is in pain. BP-PCS presents three major domains: helplessness, magnification, and rumination regarding pain. The scores of each one of the three domains are given by the sum of the corresponding items (magnification 6, 7, and 13; rumination 8-11; and helplessness $1-5$ and 12). Items are rated on a 5-point Likert scale, grading both intensity and frequency information. Results are computed by summing all items on the questionnaire and total scores range from $0-52$.

Depressive symptoms were assessed by the Beck Depression Inventory II, adapted to Brazilian Portuguese ${ }^{24}$, which is an instrument recommended by the Initiative on Methods, Measurement, and Pain Assessment in Clinical Trials since its psychometric properties are excellent and it is widely used in clinical pain research.

The State-Trait Anxiety Inventory (STAI) is a commonly used tool to measure trait and state anxiety ${ }^{25}$. It is often used in the research field to measure via self-report the presence and severity of current symptoms of anxiety and a generalized propensity to be anxious. A short version of the inventory, STAI-SV, was used in the present study ${ }^{26}$.

Sleep quality was evaluated using the Brazilian Portuguese version of the Pittsburgh Sleep Quality Index ${ }^{27}$, which has good psychometric properties, with a reliability coefficient (Cronbach's $\alpha$ ) of 0.82 .

Pain intensity was evaluated through a visual analogue scale (VAS), a common and easy method to assess pain level. VAS results were converted to 0 (no pain) to 10 (the worst pain possible). Researchers assessed the use of analgesics, defined by the average amount of analgesics used per week during the last month.

\section{Statistical analysis}

Descriptive and inferential statistics were used. After exploring data distribution, using Kolmogorov-Smirnov and Shapiro-Wilk tests, as well as inspection of histogram, we verified that the main outcomes did not meet parameters of normal distribution. Nevertheless, after comparing parametric and non-parametric approaches for group comparison and repeated-measures comparisons, analyses yielded the same results. Therefore, we used parametric descriptive (mean, standard deviation) and inferential analyses (analyses of variance [ANOVAs]). Due to several comparisons implemented, significance value was corrected according to the Bonferroni post-hoc test for intra-group comparisons and the Games-Howell post-hoc test for inter-group comparisons due to different sample sizes and violation of homogeneity. Pearson's correlations were used to verify the relation between pain catastrophizing and other clinical measures.

\section{RESULTS}

\section{General Characteristics of the Samples}

Table 1 presents sociodemographic and health aspects of the samples. All variables were significantly different between groups $(p<0.05)$, especially for the clinical aspects (depression, anxiety, sleep quality and pain level) for the healthy subjects (HS) group, which have significantly fewer or none symptoms.

\section{Comparisons of Pain Catastrophizing Scores Between Chronic Pain Syndromes}

By means of one-way ANOVAs considering pain catastrophizing scores as dependent variables, there were significant differences between the studied groups, which can be noted in Table 2. Figure 1 shows pairwise comparisons between every two groups as assessed by t-tests. The results suggest 
Table 1: Sociodemographic and health data of patients with chronic pain syndromes and healthy subjects (total $n=251)$.

\begin{tabular}{lcccccc}
\hline & HS & FM & MPS & EM & CTTH & KOA \\
& $\mathbf{n = 9 3}$ & $\mathbf{n = 4 0}$ & $\mathbf{n = 2 5}$ & $\mathbf{n = 3 3}$ & $\mathbf{n = 3 3}$ & $\mathbf{n = 2 7}$ \\
\cline { 2 - 7 } & & \multicolumn{2}{c}{ Mean (SD) } \\
\hline Age (years) & $30.4(9.6)$ & $49.6(9.8)$ & $46.0(13.3)$ & $37.6(6.0)$ & $40.2(10.8)$ & $64.7(7.8)$ \\
Years of study & $16.6(3.3)$ & $10.7(3.6)$ & $12.8(3.5)$ & $10.7(2.8)$ & $14.2(3.5)$ & $10.3(5.6)$ \\
BMI (m/kg $\left.{ }^{2}\right)$ & $24.0(4.1)$ & $22.6(4.1)$ & $20.7(3.8)$ & - & $19.7(2.7)$ & - \\
BDI-II total & $7.2(7.8)$ & $26.4(11.6)$ & $13.7(9.5)$ & - & - & $10.0(7.2)$ \\
STAI - State & $25.9(6.3)$ & $33.9(6.9)$ & $27.3(8.3)$ & - & - & - \\
STAI - Trait & $22.3(5.0)$ & $60.1(10.6)$ & - & - & - \\
PSQI & $3.1(2.0)$ & $11.8(4.2)$ & $18.9(7.4)$ & $17.2(7.2)$ & $17.1(6.8)$ & - \\
VAS - pain & $0.4(1.1)$ & $7.3(1.6)$ & $5.8(3.2)$ & $7.1(2.8)$ & $5.9(1.5)$ & $5.4(2.5)$ \\
\hline
\end{tabular}

HS = healthy subjects; FM = fibromyalgia; MPS = myofascial pain syndrome; EM = endometriosis; CTTH = chronic tensional type headache; $\mathrm{KOA}=$ knee osteoarthritis; $\mathrm{BMI}$ = body mass index; BDI-II = Beck Depression Inventory; STAI = State Trait Anxiety Inventory; PSQI = Pittsburgh Sleep Quality Index; VAS = Visual Analogue Scale; SD = standard deviation.

Table 2: Descriptive and comparative statistics for pain catastrophizing measures according to the groups of chronic pain syndromes and healthy subjects (total $n=251$ ).

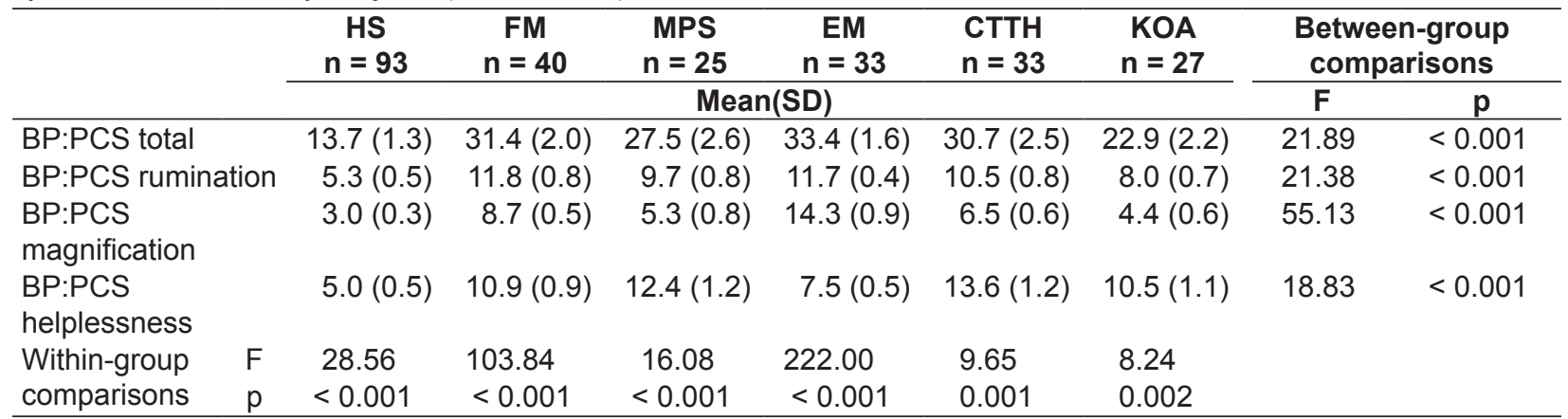

One-way analyses of variance (ANOVAs) were used for between-groups tests, and repeated-measures ANOVAs were used for within-group tests (the last included only the dimensions rumination, magnification and helplessness). HS = healthy subjects; FM = fibromyalgia; MPS = myofascial pain syndrome; $\mathrm{EM}=$ endometriosis; $\mathrm{CTTH}=$ chronic tensional type headache; $\mathrm{KOA}=$ knee osteoarthritis; $\mathrm{BP}: \mathrm{PCS}=\mathrm{Brazilian}$ Portuguese Pain Catastrophizing Scale; SD = standard deviation.

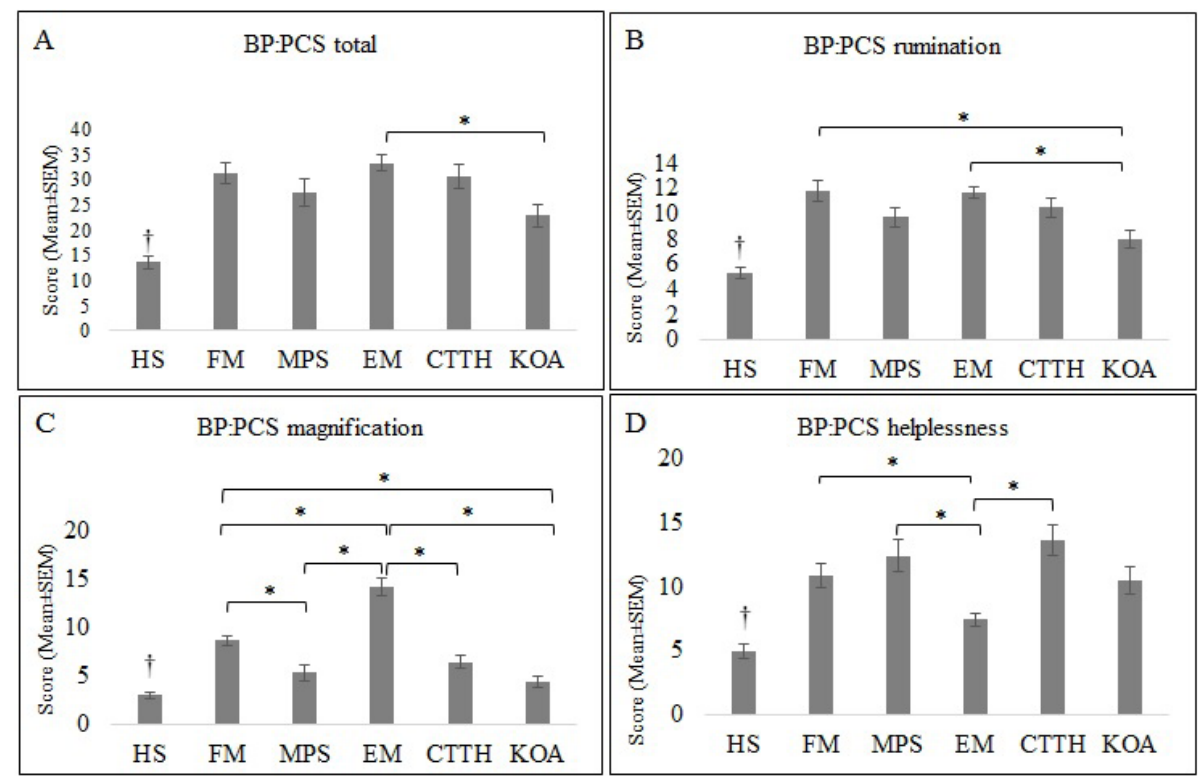

Figure 1: Differences between groups for the scores of pain catastrophizing. HS = healthy subjects; FM = fibromyalgia; MPS = myofascial pain syndrome; EM = endometriosis; CTTH = chronic tensional type headache; KOA = knee osteoarthritis; BP:PCS = Brazilian Portuguese Pain Catastrophizing Scale; SEM = standard error of the mean. ${ }^{*}=$ indicates significant differences $(p<0.05)$, according to Games-Howell post-hoc tests. $\dagger=$ indicates significant differences $(p<0.01)$, according to Games-Howell post-hoc tests between HS and all the other groups, except for magnification scores, in which HS, MPS and KOA patients were equivalent, and helplessness, in which HS and EM were equivalent. 
all measures of pain catastrophizing differ between groups. These differences were not only evident for the comparison of HS and the clinical groups, but also differ between clinical samples. For the total score (Figure $1 \mathrm{~A}$ ), patients with EM had significantly more catastrophizing thoughts than those with KOA. More interestingly were the dimensions of pain catastrophizing (Figure 1B, C and D), which showed significant differences in more than two groups, specially magnification scores, since these scores were able to discriminate, in a particular way, all the clinical samples (Figure 1C).

\section{Comparisons Between Rumination, Magnification and Helplessness Dimensions of Pain Catastrophizing}

In order to investigate whether the studied groups had a more salient prevalent aspect of pain catastrophizing, we analyzed the scores of the subscales of BP:PCS using within-subjects tests. The scores were significant different in all groups, as presented in Table 1. We plotted pairwise comparisons in Figure 2, considering post-hoc tests. We observed differences between rumination, magnification and helplessness scores in all groups, suggesting the characteristics of pain catastrophizing are distinct according to the pain disorder.

\section{Relations Between Pain Catastrophizing and Other Clinical Measures}

Table 3 presents correlations between pain catastrophizing scores and depression, anxiety, sleep quality and pain levels for the clinical groups only. We decided to not include the HS group in order to understand how these measures are correlated in chronic pain syndromes. Not every patient had all the clinical measures (except for the BP:PCS), so we indicate the $\mathrm{n}$ included in each correlation performed. Accordingly, all clinical measures are positively correlated with pain catastrophizing as a global score, as well as its dimensions (rumination, magnification and helplessness), suggesting that the more clinical symptoms reported, the more catastrophic thoughts the patients present in relation to pain.

\section{DISCUSSION}

The between-group and within-group comparisons point out to the importance of paying attention to the dimensions of pain catastrophizing according to pain syndrome, also indicating that this may not be a unitary concept. In relation to rumination, we concluded that KOA patients were less catastrophic than FM and EM patients but more catastrophic than HS. Therefore, it could be observed that KOA patients have an intermediary level of rumination. Similarly,

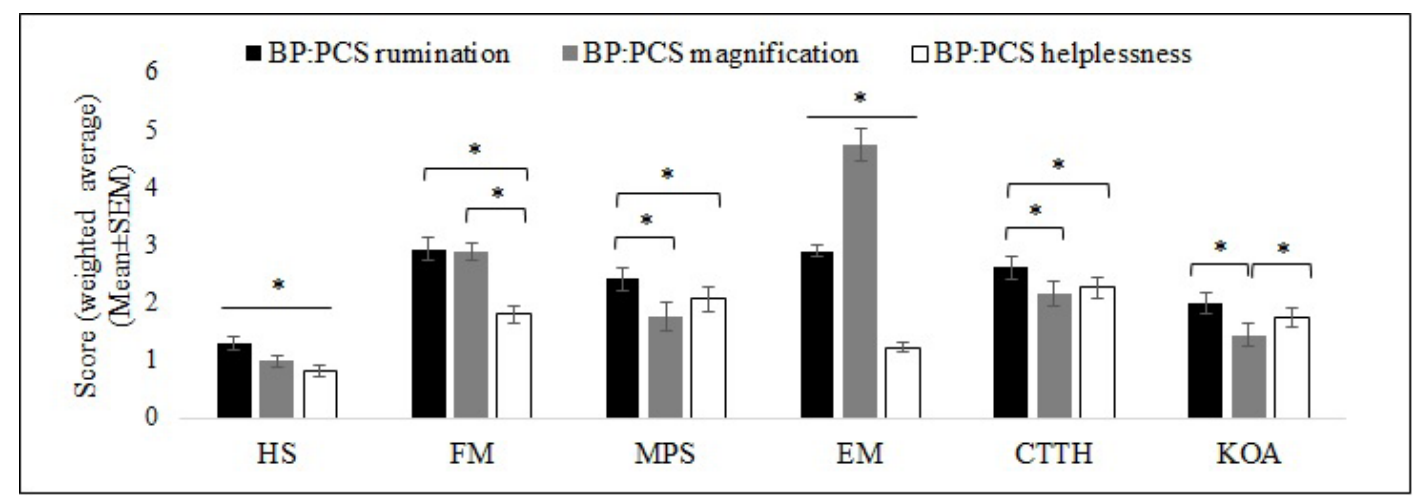

Figure 2: Differences within groups for the weighted (standardized) scores of rumination, magnification and helplessness of the BP:PCS. HS = healthy subjects; FM = fibromyalgia; MPS = myofascial pain syndrome; EM = endometriosis; $\mathrm{CTTH}=$ chronic tensional type headache; $\mathrm{KOA}=$ knee osteoarthritis; $\mathrm{BP}: \mathrm{PCS}=$ Brazilian Portuguese Pain Catastrophizing Scale; SEM = standard error of the mean. ${ }^{*}=$ indicates significant differences $(p<0.05)$, according to paired comparisons with Bonferroni correction.

Table 3: Correlations between pain catastrophizing and depression, anxiety, sleep quality and pain level.

\begin{tabular}{lccccc}
\hline & BDI-II & STAI State & STAI Trait & PSQI & VAS pain \\
& $\mathbf{n}=\mathbf{9 2}$ & $\mathbf{n = 6 5}$ & $\mathbf{n = 4 0}$ & $\mathbf{n = 1 3 1}$ & $\mathbf{n = 1 5 1}$ \\
\hline BP:PCS total & $0.70^{* *}$ & $0.50^{* *}$ & $0.55^{* *}$ & $0.33^{* *}$ & $0.35^{* *}$ \\
BP:PCS rumination & $0.73^{* *}$ & $0.49^{* *}$ & $0.59^{* *}$ & $0.25^{* *}$ & $0.35^{* *}$ \\
BP:PCS magnification & $0.68^{* *}$ & $0.49^{* *}$ & $0.42^{* *}$ & $0.21^{*}$ & $0.34^{* *}$ \\
BP:PCS helplessness & $0.52^{* *}$ & $0.39^{* *}$ & $0.47^{* *}$ & $0.32^{* *}$ & $0.22^{* *}$ \\
\hline
\end{tabular}

BP:PCS = Brazilian Portuguese Pain Catastrophizing Scale; BDI-II = Beck Depression Inventory; STAI = State Trait Anxiety Inventory; $P S Q I=$ Pittsburgh Sleep Quality Index; VAS = Visual Analogue Scale. ${ }^{*} p<0.05$; ${ }^{*} p<0.01$; all based on Pearson $r$ index. 
females with EM probably had an intermediary level of helplessness; although less catastrophic than HS, they did not reach the higher levels as for MPS, CTTH and FM patients. Taken together, these data indicate rumination would be more salient for $\mathrm{KOA}$ patients, whereas helplessness is more relevant in patients with EM.

Conversely, EM participants magnify their pain perception to a very high level, above FM, MPS, CTTC and KOA patients. Moreover, EM was the only syndrome to present more catastrophic thoughts of magnification than rumination and helplessness. Previous studies have demonstrated that pain catastrophizing predicted the persistence of pain in EM patients ${ }^{28}$. Here, we suggest magnification and exacerbation of pain would be the major problem these patients go through. In relation to MPS and CTTH, interestingly, there were no significant differences between these disorders in any aspect of pain catastrophizing. In addition, for both of them, rumination is the most salient catastrophizing factor. This may suggest that psychological and cognitive aspects of these two syndromes are similar. In fact, other authors ${ }^{29}$ discuss the presence of tender points, which characterize MPS, in patients with tension-type headache, contributing to its chronification and to the establishment of central sensitization syndromes.

Pain magnification was the best predictor of group differences in our sample. Interestingly, EM patients had the highest levels, followed by FM patients, who had more dysfunctional magnification than MPS, CTTH and KOA groups, and lastly, patients with CTTH, who had more pain magnification thoughts than $\mathrm{HS}$, as well as KOA and MPS patients. In relation to FM patients, they presented a particular catastrophizing characteristic, considering helplessness was significantly lower compared to rumination and magnification, but was not a salient aspect in distinguishing these patients from those of the other clinical groups. Our research group have found that serum S100b protein and brain derived neurotrophic factor (BDNF) was associated with lower pressure-pain thresholds ${ }^{30}$, indicating a role a central sensitization FM patients. In our previous research, pain catastrophizing scores were similar to the present study (mean $=30.73$ ). Another study found a significant relation between cortical excitability and pain catastrophizing was reported in MPS ${ }^{17}$. Taken together, these findings suggest that central nervous system physiological aspects (such as electrophysiological neuronal membrane state and neuroplasticity elements) may influence the level of catastrophizing. Patients with dysfunctional physiological state, especially when this persists over the time, becoming a dispositional characteristic, may be more vulnerable to catastrophizing thoughts.
It should be pointed out that further causal studies need to test this hypothesis.

Moreover, we may not rule out the possibility that other characteristics, such as depression and anxiety, have an important influence on these differences. As our data also indicate, pain catastrophizing is closely related to clinical measures. Some of the correlations were moderate to strong (especially when one considers depression and anxiety). There is a debate in the literature with regard to pain catastrophizing being a separate element or a component of depression and anxiety symptoms ${ }^{31}$. Although there is no final conclusion in this matter, our findings highlight the importance of considering pain catastrophizing thoughts for predicting psychological aspects in chronic pain patients, including sleep quality. These thoughts may indicate: how cognitive aspects related to patient's pain are structured; the evolution of the disorder and how pain deteriorates patient's daily activities; and how pain management and treatment strategies impact on patient's general health condition. The negative consequences of catastrophic thinking in functional aspects of patient's daily activities may be indexed by the association we found with sleep quality. Although we must keep in mind the correlational aspect of this study and that sleep is only one of the basic daily functions, pain, sleep disturbance and catastrophic thoughts sum up their effects and may deteriorate the patient's general functionality ${ }^{32}$. The relation of pain catastrophizing with pain level was also expected, although weak correlations were found here. In fact, catastrophizing thinking is associated with a distorted cognition with pessimistic beliefs about pain and is mainly regarded as a tendency or personality trait ${ }^{18}$, whereas pain levels assessed on a specific day may fluctuate greatly according to physiological, affective and social factors. In sum, although other clinical aspects are associated with pain catastrophizing, the present investigation aimed to characterize the pain catastrophizing of patients with painful syndromes using the theoretical approach of Sullivan et al. ${ }^{33}$. Other limitations are related to the lack of assessments of depression, anxiety and sleep quality in some of the syndromes, due to data was extracted from independent clinical trials, making it difficult to build a complete model so far.

\section{CONCLUSIONS}

This cross-sectional exploratory study investigated the association of chronic pain syndromes from diverse etiologies and distinct aspects of pain catastrophizing. Patients and healthy volunteers responded to the BP:PCS and to other clinical assessment instruments. HS clearly differ from clinical groups for all measures, 
as expected. Magnification was the most sensitive measure to differentiate the groups, although helplessness, rumination and even the total score were particularly associated with some of the groups. These findings have implications for the theoretical aspects of pain catastrophizing, corroborating its multidimensional structure, and for practical issues, serving well for researchers and clinicians who need to consider cognitive and emotional aspects of pain perception in order to implement more appropriate interventions.

\section{Acknowledgements}

This study was funded by the following institutions: Coordination for the Improvement of Higher Education Personnel (Coordenação de Aperfeiçoamento de Pessoal de Nível Superior, CAPES) (post-doctoral and doctoral scholarships); National Council for Scientific and Technological Development (Conselho Nacional de Desenvolvimento Científico e Tecnológico, CNPq) (fellowship of research productivity and post-doctoral scholarships); Funding for Research Incentive (Fundo de Incentivo à Pesquisa, FIPE/HCPA) in all randomized clinical trials; Brazilian Innovation Agency (Financiadora de Estudos e Projetos, FINEP) process number - 1245/13; Foundation for the Support of Research at Rio Grande do Sul (Fundação de Amparo à Pesquisa do Estado do Rio Grande do Sul, FAPERGS); Graduate Program in Medical Sciences at the School of Medicine of Universidade Federal do Rio Grande do Sul and Graduate Research Group at the Hospital de Clínicas de Porto Alegre (material support).

\section{Conflicts of Interest}

The authors declare no conflicts of interest.

\section{REFERENCES}

1. Cimmino MA, Ferrone $C$, Cutolo $\mathrm{M}$. Epidemiology of chronic musculoskeletal pain. Best Pract Res Clin Rheumatol. 2011;25(2):17383. http://dx.doi.org/10.1016/j. berh.2010.01.012. PMid:22094194.

2. Skootsky SA, Jaeger B, Oye RK. Prevalence of myofascial pain in general internal medicine practice. West J Med. 1989;151(2):157-60. PMid:2788962.

3. Fayaz A, Croft $P$, Langford RM, Donaldson LJ, Jones GT. Prevalence of chronic pain in the UK: a systematic review and meta-analysis of population studies. BMJ Open. 2016;6(6):e010364. http://dx.doi. org/10.1136/bmjopen-2015-010364. PMid:27324708.

4. Staud R, Robinson ME, Price DD. Temporal summation of second pain and its maintenance are useful for characterizing widespread central sensitization of fibromyalgia patients. J Pain. 2007;8(11):893901. http://dx.doi.org/10.1016/j. jpain.2007.06.006. PMid:17681887.

5. Xu Y-M, Ge HY, Arendt-Nielsen L. Sustained nociceptive mechanical stimulation of latent myofascial trigger point induces central sensitization in healthy subjects. $J$ Pain. 2010;11(12):1348-55. http://dx.doi. org/10.1016/j.jpain.2010.03.010. PMid:20451466.
6. Garland EL. Pain processing in the human nervous system: a selective review of nociceptive and biobehavioral pathways. Prim Care. 2012;39(3):561-71. http://dx.doi. org/10.1016/j.pop.2012.06.013. PMid:22958566.

7. Schur EA, Afari N, Furberg $\mathrm{H}$, Olarte M, Goldberg J, Sullivan PF, et al. Feeling bad in more ways than one: comorbidity patterns of medically unexplained and psychiatric conditions. J Gen Intern Med. 2007;22(6):818-21. http://dx.doi. org/10.1007/s11606-007-0140-5. PMid:17503107.

8. Chapman CR, Nakamura Y. A passion of the soul: an introduction to pain for consciousness researchers. Conscious Cogn. 1999;8(4):391422. http://dx.doi.org/10.1006/ ccog.1999.0411. PMid:10600241.

9. Edwards RR, Goble L, Kwan A, Kudel I, McGuire L, Heinberg $\mathrm{L}$, et al. Catastrophizing, pain, and social adjustment in scleroderma: relationships with educational level. Clin J Pain. 2006;22(7):63946. http://dx.doi.org/10.1097/01. ajp.0000210918.26159.94. PMid:16926580.

10. Geisser ME, Robinson ME, Keefe FJ, Weiner ML. Catastrophizing, depression and the sensory, affective and evaluative aspects of chronic pain. Pain. 1994;59(1):79-83. http://dx.doi.org/10.1016/03043959(94)90050-7. PMid:7854806.

11. Sullivan MJ, Rodgers WM, Kirsch I. Catastrophizing, depression and expectancies for pain and emotional distress. Pain. 2001;91(1-2):147-54. http://dx.doi.org/10.1016/S03043959(00)00430-9. PMid:11240087.

12. Beck A. Cognitive therapy and the emotional disorders. New York: New York Meridian; 1976.

13. Beck A, Emery G, Greenberg R. Anxiety disorders and phobias: a cognitive perspective therapy. New York: Basic Books; 1985.

14. Sullivan MJ, Lynch ME, Clark AJ. Dimensions of catastrophic thinking associated with pain experience and disability in patients with neuropathic pain conditions. Pain 2005;113(3):310-5. http://dx.doi. org/10.1016/j.pain.2004.11.003. PMid:15661438.

15. Sullivan M, Thorn B, Haythornthwaite JA, Keefe F, Martin M, Bradley LA, et al. Theoretical perspectives on the relation between catastrophizing and pain. Clin J Pain. 2001;17(1):52-64. http://dx.doi. org/10.1097/00002508-20010300000008. PMid:11289089.

16. Sullivan M, Bishop SR, Pivik J. The pain catastrophizing scale: development and validation. 
Psychol Assess. 1995;7(4):524-32. http://dx.doi.org/10.1037/10403590.7.4.524.

17. Volz MS, Medeiros LF, Tarragô MG, Vidor LP, Dall'Agnol L, Deitos A, et al. The relationship between cortical excitability and pain catastrophizing in myofascial pain. J Pain. 2013;14(10):1140-7. http://dx.doi. org/10.1016/j.jpain.2013.04.013. PMid:23810270.

18. Sullivan MJ, Thorn B, Haythornthwaite JA, Keefe F, Martin M, Bradley LA, et al. Theoretical perspectives on the relation between catastrophizing and pain. Clin J Pain. 2001;17(1):52-64. http://dx.doi. org/10.1097/00002508-20010300000008. PMid:11289089.

19. Altman R, Asch E, Bloch D, Bole G, Borenstein D, Brandt K, et al. Development of criteria for the classification and reporting of osteoarthritis: Classification of osteoarthritis of the knee. Arthritis Rheum. 1986;29(8):1039-49. http:// dx.doi.org/10.1002/art.1780290816. PMid:3741515.

20. Fernandes MI. Tradução e validação do questionário de qualidade de vida específico para osteoartrose WOMAC (Western Ontario McMaster Universities) para a língua portuguesa. São Paulo: Universidade Federal de São Paulo; 2003.

21. Olesen J, Steiner TJ. The International classification of headache disorders. J Neurol Neurosurg Psychiatry. 2004;75:808-11.

22. Wolfe F, Clauw DJ, Fitzcharles MA, Goldenberg DL, Katz RS, Mease $P$, et al. The American College of Rheumatology preliminary diagnostic criteria for fibromyalgia and measurement of symptom severity. Arthritis Care Res. 2010;62(5):600-10. http://dx.doi.org/10.1002/acr.20140. PMid:20461783.

23. Sehn F, Chachamovich E, Vidor LP, Dall-Agnol L, Souza ICC, Torres ILS, et al. Cross-cultural adaptation and validation of the Brazilian Portuguese version of the pain catastrophizing scale. Pain Med. 2012;13(11):1425-35. http://dx.doi.org/10.1111/j.15264637.2012.01492.x.

24. Gomes-Oliveira MH, Gorenstein $\mathrm{C}$, Lotufo F No, Andrade LH, Wang YP. Validation of the Brazilian Portuguese Version of the Beck Depression Inventory-II in a community sample. Rev Bras Psiquiatr. 2012;34(4):38994. http://dx.doi.org/10.1016/j. rbp.2012.03.005. PMid:23429809.

25. Spielberger CD, Gorsuch RL, Lushene R, Vagg PR, Jacobs GA. Manual for the State-Trait Anxiety Inventory (STAI). Palo Alto (CA): Consulting Psychologists Press; 1983.

26. Kaipper MB, Chachamovich E, Hidalgo MPL, Torres ILS, Caumo W. Evaluation of the structure of Brazilian State-Trait Anxiety Inventory using a Rasch psychometric approach. J Psychosom Res. 2010;68(3):223-33. http://dx.doi. org/10.1016/j.jpsychores.2009.09.013. PMid:20159207.

27. Bertolazi AN, Fagondes SC, Hoff LS, Dartora EG, Miozzo ICS, Barba MEF, et al. Validation of the Brazilian Portuguese version of the Pittsburgh Sleep Quality Index. Sleep Med. 2011;12(1):70-5. http://dx.doi. org/10.1016/j.sleep.2010.04.020. PMid:21145786.
28. Martin CE, Johnson E, Wechter ME, Leserman J, Zolnoun DA. Catastrophizing: a predictor of persistent pain among women with endometriosis at 1 year. Hum Reprod. 2011;26(11):3078-84. http:// dx.doi.org/10.1093/humrep/der292. PMid:21900393.

29. Bendtsen L, Fernández-de-la-Peñas C. The role of muscles in tensiontype headache. Curr Pain Headache Rep. 2011;15(6):451-8. http://dx.doi. org/10.1007/s11916-011-0216-0. PMid:21735049.

30. Zanette SA, Dussan-Sarria JA, Souza A, Deitos A, Torres IL, Caumo W. Higher serum S100B and BDNF levels are correlated with a lower pressurepain threshold in fibromyalgia. Mol Pain. 2014;10:46. http://dx.doi. org/10.1186/1744-8069-10-46. PMid:25005881.

31. Leung L. Pain catastrophizing: an updated review. Indian J Psychol Med. 2012;34(3):204-17. http://dx.doi. org/10.4103/0253-7176.106012. PMid:23441031.

32. Flink IK, Linton SJ. Pain, sleep and catastrophizing: The conceptualization matters: Comment on Wilt JA et al. "A multilevel path model analysis of the relations between sleep, pain, and pain catastrophizing in chronic pain rehabilitation patients. Scand J Pain. 2016;10:119-21. PMid:28361762.

33. Sullivan MJ, Lynch ME, Clark AJ. Dimensions of catastrophic thinking associated with pain experience and disability in patients with neuropathic pain conditions. Pain. 2005;113(3):310-5. http://dx.doi. org/10.1016/j.pain.2004.11.003. PMid:15661438. 